\title{
PYURIA
}

\author{
By JOHN EVERIDGE, F.R.C.S. \\ (Urologist and Lecturer on Urology, King's College Hospital.)
}

\section{DEFINITION OF PYURIA.}

By this term it is understood that there is a discharge of leucocytes which escape during the passage of urine in sufficient numbers to constitute pus. Leucocytes may be found in the urine commonly enough in subjects where no evidence whatsoever of disease is apparent. Cuthbert Dukes ("British Medical Journal," I928, i, 39I) limits the term in adults to those cases which show more than Ioo cells per c.mm. The incidence of leucocytes in the urine of apparently healthy children was investigated by J. N. Cummings (" British Medical Journal, I93I, i); he found that 55 per cent. of the girls of his series up to II years of age yielded counts from 5 to 64 cells per c.mm., whereas only 3.4 per cent. of the boys showed any cells at all. Cummings found that urine became opalescent in the presence of 200 cells per c.mm.; only the microscope can detect a lower content. Accepting Dukes' figure as "pyuria," it is to be assumed that this is a pathological state and is the indication of an inflammation, almost certainly of a bacterial nature, not only from any part of the excretory tract, from glomerulus to external meatus, but from the genital adnexa within, as well as from the external genitalia.

\section{DETERMINATION OF PYURIA-NECESSARY PRECAUTIONS.}

For the correct determination of the existence of pyuria, collection of a specimen for examination by catheter is essential in the female, to exclude vulval contamination. In the male, if the prepuce be retracted and the meatus cleansed, a specimen passed naturally will suffice, in the absence of urethritis. Where urethral inflammation is suspected the "two glass" test is generally adequate, but more precise information is supplied by a preliminary urethral wash-out by the Janet method.

\section{TYPES OF PYURIA.}

Since an anatomically perfect urinary tract is prone to spontaneous recovery from inflammations, except in the case of tuberculous disease, pyuria tends to be short lived; persistence should give reason to suspect a lasting source of supply of organisms outside the tract, e.g., from the bowel or genital adnexa, or such mechanical or structural defects as will cause lowered resistance or urinary stagnation. Broadly, we may expect to find, where inflammations occur, (a) acute attacks tending to rapid and spontaneous recovery, if the tract is perfect structurally, (b) relapsing inflammations, where a defective bowel or the type of organism within it are responsible, (c) chronic inflammation, with anatomical imperfections, e.g., stones, growths, or an unrelieved mechanical obstruction, congenital or acquired.

A focus of sepsis alongside or outside the urinary tract, and which persists because it has never been properly drained, and intermittently or continuously discharges its infecting contents into the urinary tract, as for instance, a para-colic 
abscess of diverticulitis or a prostatic abscess, may cause either relapsing or chronic pyuria. The surgery of pyuria to-day is the investigation to discover the origin and cause of the factors predisposing to bacterial inflammation. Modern methods provide almost unlimited facilities for unravelling these problems and, as the means of diagnosis has developed, surgical treatment has become more standardized and precise. It may safely be said that painstaking diagnosis is the soul of modern urology.

\section{ETIOLOGY.}

In discussing the causes of pyuria it is proposed to give a short description of the types as they are prone to occur at the several periods of life before consideration of the standard methods of investigation, which are, for the most part, common to all. Some especial treatments for each will be dealt with under the several headings, whilst general principles of urinary disinfection will be reserved for the concluding remarks. To catalogue a complete list of the causes of pyuria would be to recite a list of specimens of any well-equipped urological Museum, and this would, by no means, be comprehensive; only the more common types come within the scope of this paper.

\section{First ro Years of Life.}

Pyelo-cystitis due to $B$. coli and Staphylococcus Infection. The acute B. coli variety may be of intestinal origin, or, in females, from an ascending infection from the vulva. The chronic or relapsing variety may result from, or be associated with, calculous disease of the kidneys, ureters and bladder, and this is especially prone to occur in children who have been subjected to the prolonged recumbent posture for treatment of an orthopædic condition, especially tubercle of the spine or hip. Another frequent predisposing cause is a developmental defect in any part of the urinary tract, amongst the most common of which are hydronephrosis from misplaced renal vessels obstructing the pelvic outlet, and double pelvis or ureter. Reference to this subject in greater detail is made by Mr. Addison (p. 380). A diverticulum in the bladder situated near the ureter orifice may so obstruct the duct as to cause hydroureter and hydronephrosis, thus producing a soil suitable for infection. Mal-development of the genitals, ectopia vesicæ, epi- and hypospadias, allow access of organisms from without and sepsis, sooner or later, supervenes.

Foreign bodies may find their way into the bladder in either sex, but this cause of sepsis is more liable to occur in the second decade. In infants, especially males, an ascending infection from the urethra may arise from infection from napkins. Such may commence as an ulcer at the meatus from irritation of the napkin contaminated with alkalinizing germs from the bowel. In these cases the condition will be readily cured if the napkins are frequently soaked in boracic lotion.

Pyuria from urinary tuberculosis is rare in the first decade.

ro-20 Years of Age.

The developmental abnormalities of the kidney, ureter or bladder may not manifest themselves by infection until this or later periods of life. 
In this decade the incidence of tubercle increases and hence must be borne in mind whilst searching for a diagnosis. B. coli or other organisms may be present as a superadded infection, their presence misleading us and causing one of the well-known pitfalls in diagnosis. Cystoscopy will generally reveal the fundamental malady by discovering a typical lesion and stimulating the search for the acidfast organism.

Attacks of idiopathic pyelocystitis, due to B. coli with, perhaps, enterococci, are not uncommon at the age of puberty; they usually are readily cured by urinary antiseptics, but should be treated during the early period of acute symptoms by sufficient amounts of alkali to procure an alkaline urine. Pot. Cit. may be given, even up to I drachm doses, six-hourly.

Staphylococcus infection should always arouse the suspicion of stone, and if remaining in the urine beyond the period of a normal acute attack of inflammation lasting some IO-I4 days, a radiographic examination should be made.

Foreign bodies (wax and tapers) in the bladder or urethra of adolescent males may explain some otherwise unaccountable causes of cystitis. These are not so uncommon as might be imagined, and a good many examples have presented themselves in the practice of the author.

\section{0-30 Years of Age.}

Gonorrhœea and its complications will account for a high percentage of examples of pyuria at this age; It is not intended to discuss venereal disease in this paper. At this age, in both sexes, urinary tuberculosis should be written in red. Every case of pyuria which has not commenced with acute symptoms or fails to respond to simple measures and is probably not gonorrhœal, and especially if the pyuria is known to have persisted for a considerable time, should be regarded with grave suspicion and every effort made to exclude tuberculosis. Pus in a sterile urine which is usually acid, should be held as highly suspicious, and cystoscopy, etc., at once advised. In the absence of T.B. in suspicious cases, the guinea pig injection test should be carried out.

At this age, in females, especially in the newly-wed, acute B. coli pyelo-cystitis is very prone to occur. Why it should be so common in the early weeks of married life is difficult to explain; support is given to the theory popular at the present time that, in the female, a high percentage of cases of B. coli infection in the urinary tract are of genital origin. The association of cervicitis, especially at a later age, is well recognized, and the cervix uteri is doubtless a source of lymphatic distribution to the urinary organs.

In this third decade we shall meet the majority of our cases of pyuria from pyelitis of pregnancy, a complication of pregnancy of which we can boast, fortunately, of having obtained considerable measure at the present time, and which but seldom leads to so serious a climax as to necessitate premature termination of gestation. The recognition of the necessity for pelvic drainage, and of its attainment with the aid of a ureteric catheter, as well as the properly regulated administration of alkalis or of hexamine, have gone far to deprive this form of pyelitis of its erstwhile difficulties and dangers. Hexamine administered intravenously (see later) has been proved satisfactory. 


\section{0-50 Years of Age.}

In the male, pyuria is common from the later complications of gonorrhœa, strictures, chronic prostatitis, etc. Stones in the kidney are beginning to become more frequent in both sexes and in the bladder in the male. These may be the cause or effect of sepsis and therefore are often associated with pyuria, the recognition of which may be the first indication of the presence of a calculus. Tuberculosis is still to be borne in mind, but is becoming less probable at this age. In the female, chronic or relapsing B. coli pyelo-cystitis is extremely common, often as an aftermath of pregnancy, or associated with chronic endometrial infection or bowel disorders.

\section{0-70 Years of Age.}

We have now arrived at the period when the male has to bear more than his share of urinary affections, for enlargement of the prostate comes into the picture, and is frequently complicated by pyuria from cystitis. Pyuria with the enlarged prostate is not always easy to explain; it is not merely the result of stasis, for we are all familiar with those giant bladders distended to 2,3 or even 4 pints, where the urine remains clear as crystal, and the alternative state, where there is perhaps only one ounce of residual urine, but associated with severe cystitis and a highly purulent urine which, however, clears completely after removal of the gland. Further conditions promoting pyuria in prostate cases are stones and bladder diverticula, and, if a catheter life is resorted to, the catheter. The enlarged prostate containing embedded or subcapsular calculi is frequently associated with pyuria and a troublesome cystitis. The malignant prostate, on the other hand, does not pre-dispose to bladder sepsis; the absence of pyuria is not without value as a sign in diagnosing the malignant from the fibrous or chronic inflammatory prostate. The accompaniment of pyuria is, too, of value in the differential diagnosis of bladder tumours, but here it is the malignant growth which predisposes to sepsis; the bladder which is a suitable soil for papillomata appears to possess a higher than normal resistance to sepsis, as may be deduced from the astonishing way in which sterility remains in spite of the repeated insults of cystoscopic diathermy. In several cases in my series I have carried out this treatment more than twenty times without signs of sepsis; soiling must have occurred, but no infection. In a majority of cases of vesical carcinoma I have found pyuria before a cystoscope or other instrument had been passed.

\section{Unusual Causes.}

A. Ulceration of Bladder. Ulcers of the bladder as a cause of purulent urine we hear much of but seldom see. They appear to be far more frequent in the practice of the less experienced observers. The ulcers I most commonly see, and they are definite rarities, are tuberculous ulcers, malignant ulcers, and small ulcers somewhat resembling peptic ulcers on the buccal mucous membrane. The last may be the accompaniment of a simple pyogenic infection or of a cystitis in which no pus or causative organisms can be found; tuberculosis as the cause may be excluded by the guinea pig test. Radium ulcers, resulting from gynæcological applications, are not uncommon and usually cause a pyuria.

B. Vesical Fistula. A communication between the bladder and skin or any hollow viscus must necessarily lead to infection. Excluding vesico-cutaneous 
fistulæ, the majority exist between the bladder and colon or rectum, and may be congenital or acquired. At any age wounds or inflammatory processes can occur as the primary cause and, in elderly subjects, diverticulitis with abscess formation, or malignant neoplasm extending from the bowel to the bladder, or in the reverse direction, may explain the existence of persisting pyuria. With the exception of examples due to carcinomata, the condition usually clears up rapidly where the source of infection is removed as, for instance, after colostomy for a diverticulitis fistula. The power of resistance and recovery of the bladder is well proved in many of these cases, for cystoscopy so frequently reveals a mucous membrane in an almost normal state, in spite of persisting re-infection or recent inflammation. A history of flatus or fæces passed with the urine may generally be obtained in these cases and suggests the probable cause of the pyuria.

\section{EXAMINATION OF A CASE OF PYURIA.}

In many instances the history and symptoms may suggest the origin, but too much reliance must never be placed upon these in the diagnosis of the origin of pyuria as, in fact, in many other urinary conditions. In urinary tuberculosis, for instance, the primary pathology is in the kidney, yet, in the majority of cases, the patient has never had reason to believe, from his sensations, that this organ is at fault. In the simple pyogenic infections, too, a septic hydronephrosis or even a calculous pyonephrosis may give no symptoms, or perhaps only the symptoms of a mild cystitis. Thus, in practically every case we must subject our patients to the solemn ritual of urological investigation, seldom venturing to side-step a single link in the chain of evidence, for it is only by taking advantage of the cystoscope and ureteric catheterization, radiography and pyelography, that the causative agents in chronic or relapsing cases can be ascertained.

After the usual bedside examination, which must always include examination of the external genitals, as well as a vaginal and rectal investigation, the urine will come under consideration. After noting the colour, reaction, and specific gravity, cytological and bacteriological investigations are made. The total quantity passed during the 24 hours is often of importance, especially where the origin of the pyuria is, possibly, one or both kidneys.

Bacteriological investigation in a case under examination is easy to carry out and, in the case of tubercle, is all-important, but in many other infections too much time is spent, and often wasted, on bacteriology, when war should be waged against those predisposing factors without which the infection could never have occurred or persisted.

Cystoscopy, though of profound importance, is bound to be disagreeable to the patient and may require general anæsthesia. I, now-a-days, find intravenous pyelography so informative as to make cystoscopy often unnecessary, especially in children, and generally, therefore resort to it, anyhow in private practice, before making a direct examination of the bladder. Control radiography of the urinary tract is carried out before injection of the opaque substance, to exclude calculi. A general survey of the whole urinary tract is, by intravenous pyelography, readily obtained and the relative and absolute function of the kidneys, as well as their architecture, can be gauged with tolerable accuracy. The lead is often given as to whether, and how, to proceed with cystoscopy. Retrograde pyelography with Sod. Iodide, I3.5 per cent. solution, may be necessary to confirm a doubtful excretion 
urogram or to define the ureter with greater accuracy. Negative radiography must never be regarded as excluding stone. Many a time I have found large stones on cystoscopy and, subsequently, when performing litholopaxy, discovered calculi, though transparent to X-rays, of unusual hardness and which strained the lithotrite to its full.

In pyuria from tuberculosis we usually have occasion to employ all the most modern aids to urologicai diagnosis. In this disease, too, we must thoroughly overhaul the genital tract and, indeed, the whole patient, to be in a position to indicate the policy of treatment and the reasonable prognosis.

The apparently simple cases of $\mathrm{B}$. coli pyelitis, those everyday examples which, perhaps, are now and then accompanied by attacks of renal pain and pyrexia, are repeatedly of profound clinical and pathological interest, and have definitely become more so since the routine use of intravenous pyelography as an instrument of diagnosis. This is so from the very high incidence of gross anatomical defects found to exist in the kidney and ureter. It is no exaggeration to say that in the majority of cases of chronic pyelitis which come under observation abnormalities such as hydronephrosis, double kidneys, kinked ureters, stones, horseshoe kidneys, are found to be present. Many a case labelled " chronic cystitis", represents a cystitis rendered chronic by the constant re-infection from a kidney maintained in a state of sepsis by the predisposing influence of one of these abnormalities (see Fig. 9). It is easy to see, therefore, why the many antiseptics, vaccines, pelvic lavages, etc. ad nauseam, have all come to grave disrepute. With such architectural defects, no attack on sepsis, without operation to correct malformation or, at any rate, to ensure good drainage, can be expected to meet with success; our judgment is often severely tested to decide upon the justification for major surgery in these cases.

\section{TREATMENT.}

(a) Chronic Infections. Enough has been said to emphasize the necessity for direct attack by surgery upon the primary causative factor, nephrectomy for tubercle, removal of an aberrant artery producing hydronephrosis, prostatectomy, etc. Any defect in the gastro-intestinal apparatus must be attended to either medically or surgically as occasion demands. In the absence of definite indication for operation, where, for instance, pyelography has only shown a minor structural defect, such as a slight degree of hydronephrosis associated with a dropped kidney, or where constitutional weakness excludes operation, how are we best to attack the focal sepsis? Shall we try urinary antiseptics; and, if so, which? Are vaccines of value, or should we advise a course of ketogenic diet? From foregoing observations it is clear that there is little hope of sterilizing the fons et origo of infection in the urinary tract, where there is mechanical imperfection. It is well known that chronic cystitis is almost impossible to cure if there is chronic vesical retention from an enlarged prostate, or a stone is present: in the kidney imperfect drainage from a blocked ureter, etc. is analogous. With this knowledge any course to be adopted will be commenced with misgivings, but not without some hope for, although failing to cure, there is no doubt that the various remedies indicated lower the mass action of the infecting agents, hold the organisms in some way or other in check, and to a large extent neutralize the irritating and toxic effect of the germs. Whether hexamine is better than the various dyes which are popular to-day, can, I believe, 
only be proved by trying it in the individual case. My own experience has taught me that the B. coli carrier, who has experienced periodical flares-up, is likely to remain in better health if he continues with his daily I5 grain doses of hexamine year in year out. The dyes occasionally appear to aid in the resolution of a more acute attack, but there are disadvantages to their prolonged use, not the least of which is the staining of linen. The victim of pyelitis of pregnancy can often survive to full term without serious consequences if hexamine be commenced in the early months and continued faithfully throughout.

With the ketogenic diet, unless a pus-free and germ-free urine be obtained by the end of the second week (assuming that acidity and ketosis have been brought to the required standard) it is doubtful if this treatment will ever sterilize the urinary tract. Although failing to achieve what has been striven for, it is plain that many of the cases are markedly benefited symptomatically; especially has this been observed where cystitis with distressing frequency of micturition was the predominant symptom.

Pelvic lavage for chronic pyelitis occasionally has succeeded where all else has failed; it is conceivable that passage of the ureteric catheter is a factor promoting success, if a narrow ureter was the predisposing factor of causation. It is difficult to understand how a complicated arrangement like the pelvis and calices could be cleared of septic inflammation with the few washes that are given, and with so weak a solution as I in I0,000 silver nitrate, the strength usually employed. (Collargol, Protargol and Argyrol are also used).

In some clinics nephrostomy has been used for chronic pyelitis and pyelonephritis, a tube being passed through the renal parenchyma into the pelvis or a dilated calyx. This tube is allowed to remain some six weeks and, in favourable cases, it will be found that the sepsis has cleared itself up. Drainage in this way can only be instituted where there is some degree of hydronephrosis.

(b) Acute Infections. Primary treatment by alkalies remains the standard method of attack in the acute B. coli infections. As an alternative in this type, especially where associated with stapylococcal or streptococcal infections, mention must be made of the intravenous administration of hexamine. A solution of this substance combined with sodium salicylate and caffeine sodium salicylate, known as cylotropin, has been found of value in bringing to an end acute pyrexial attacks from urinary sepsis, but it has also proved its use, in my hands, as a means of preventing a septic reaction, where this was to be anticipated from the nature of the case under consideration. I cannot better illustrate this than by calling attention to its value in connection with trans-urethral electro-resection of the prostate. In the early days of this operation post-operative sepsis was one of the great bugbears and was recognized as a deterrent to its employment. In my first series of endoscopic resections the fear of sepsis impelled me to the frequently applied safeguard of preliminary suprapubic drainage, in cases where $I$ felt that septic complications would turn the scale against the already debilitated patient, whose constitution had been taxed to the full. I at first resorted to cylotropin where signs of septic absorption, pyrexia or rigors, had already developed. The rapid disappearance of these manifestations in several cases convinced me of the very real advantage gained by the intravenous administration of this compound of hexamine, and it appeared obvious that it would be reasonable in every case to use it prophylactically. I have resorted to this policy in my last hundred or so of endoscopic 
resections, and it is no exaggeration to state that in no single case has sepsis caused the least anxiety. Incidentally, too, there have been very few examples of epididymitis.

Similarly, in cases of stricture dilatation, where rigors, etc. ("catheter fever") are prone to occur, cylotropin injected at the time of dilatation will prevent such complications.

Representation of the advantages of this substance to my gynæcological colleagues has borne fruit, for I learn that pyelitis of pregnancy is definitely improved and even cured, and that B. coli infection after hysterectomy, etc. is often aborted and, curiously enough, the incidence of post-operative retention of urine appears to be lowered.

In regard to oral administration of the urinary antiseptics, so much has already been written of hexamine elsewhere that I make no allusion to it other than to emphasize the necessity of an acid urine and, in my experience, it acts best if given one hour after half to one drachm doses of acid sodium phosphate, three times a day, for choice the phosphate being given a.c. and the hexamine p.c. Where the urine is naturally acid the phosphate is unnecessary. Of the more recent antiseptics neotropin has appeared to be one of the best, but digestive and bowel disturbances must be watched for, and their occurrence would be the signal for its discontinuance. Methylene blue has remained a favourite substance and, further, it appears to exercise a carminative action on the bladder; it may also act in an alkaline urine.

The ketogenic-diet treatment is often left to the last resort largely, perhaps, because it is still a novelty, and the well-known methods and older medicaments demand prior rights from seniority. Further, being useless unless carried out to the minutest detail, its demands are greater and resources are more heavily taxed in every way. Experience of its failures must therefore indicate caution in advising its trial; at least a fortnight must be devoted to it, and institutional treatment has generally to be established. In my own experience the most satisfactory cases have been those of B. coli cystitis associated with great frequency and irritability; although in many the urine has not been sterilized, the symptoms have been relieved in a dramatic way, and life has again become endurable and even enjoyable to cases which appeared almost desperate enough to demand suprapubic drainage for relief.

Bladder lavage hardly calls for comment, its advantages must be familiar to all. I still place weak solutions of silver nitrate ( $\mathrm{I}$ in I2,000 in Aq. distill.) among the foremost in value; it appears to have almost a specific action upon the bladder mucous membrane. Hg. Oxycyanide ( $\mathrm{I}$ in 8,000) is a useful alternative, but even in this dilution may be too irritating. Boracic lotion, weak pot. permang. solution, or even saline, should be used for the most irritable bladders, and I am also using, in these cases, upon the advice of my friend Mr. A. R. Thompson, of Guy's Hospital, a solution of tannic acid, 5 grammes in water to Ioo ounces, with success.

The subject of pyuria entails a consideration of the majority of problems of urological pathology, introduces every method of modern investigation, endoscopic, radiological, biochemical and chemical, and calls for treatment upon the broadest possible lines, from the most refined surgical operations to the tempting of the palate of an unpalatable diet in the hands of a skilful cook. 